12

\title{
Концептуальная модель квазибессилового магнита малого объема с инерционным удержанием его торцевой части
}

\author{
(C) Г.А. Шнеерсон, А.А. Парфентьев, В.В. Титков, С.И. Кривошеев, А.Д. Лагуткина, \\ А.С. Немов, А.П. Ненашев, С.А. Шиманский
}

Санкт-Петербургский политехнический университет Петра Великого, Санкт-Петербург, Россия

E-mail: gashneerson@mail.ru

Поступило в Редакцию 1 декабря 2020 г.

В окончательной редакции 12 марта 2021 г.

Принято к публикации 12 марта 2021 г.

\begin{abstract}
Показана эффективность применения диамагнитного экрана с инерционным удержанием в неразрушаемом квазибессиловом магните малого объема (масштаба $10 \mathrm{~cm}^{3}$ ) для получения сверхсильного магнитного поля с длительностью импульса порядка $10 \mu \mathrm{s}$. Построена конфигурация магнита, рассчитаны механические напряжения в обмотке, торцевая часть которой уравновешена благодаря тому, что экран практически не смещается за время разряда. Численные расчеты показали, что механическая прочность обмотки может быть обеспечена в поле с индукцией до $100 \mathrm{~T}$. Выполнены расчеты нагрева обмотки и указаны возможности его снижения до приемлемого уровня.
\end{abstract}

Ключевые слова: квазибессиловая обмотка, неразрушаемый магнит, сильные импульсные магнитные поля, нагрев проводника.

DOI: 10.21883/PJTF.2021.11.51007.18639

Особенностью современных неразрушаемых магнитов, предназначенных для получения полей с индукцией, достигающей мегагауссного уровня, является использование равнонагруженных многослойных обмоток [1,2]. Внешний радиус такого магнита должен быть много больше внутреннего, а энергия системы - много больше энергии в его рабочей области. Например, в магните с внутренним диаметром $15.5 \mathrm{~mm}$, описанном в работе [2], для получения поля с индукцией около 90 Т используется мотор-генератор для создания поля с индукцией $36 \mathrm{~T}$ в первой и конденсаторная батарея с энергией $2.5 \mathrm{MJ}$ во второй стадии разряда. Альтернативой таким системам может быть магнит с квазибессиловой обмоткой, в которой остаточные механические напряжения могут быть снижены до значений, существенно меньших, чем магнитное давление генерируемого поля, при гораздо меньших размерах и энергии магнитной системы $[3,4]$. В таких магнитах наряду с обмоткой, разгруженной от электромагнитных сил, имеет место внешняя граница, на которую воздействует магнитное давление $P_{M}=P_{0} / A^{2}$, где $P_{0}=B_{0}^{2} /\left(2 \mu_{0}\right)-$ магнитное давление с индукцией $B_{0}$ на оси магнита, $A=R_{2} / R_{1}-$ аспектное отношение $\left(R_{1}-\right.$ внутренний, а $R_{2}-$ внешний радиус магнитной системы). Давление на бандаж, удерживающий внешнюю границу, может быть резко снижено при большом значении аспектного отношения. В работах по теории квазибессиловых магнитных систем показано, что для достижения высоких значений этого параметра при сохранении равновесия торцевой части может быть использован торцевой диамагнитный экран [5]. Его создание существенно облегчается, если магнит предназначен для получения кратковременных импульсов магнитного поля в малых объемах. В этих условиях может быть использована инерция экрана для его удержания на время разряда, что позволяет избежать разрушения обмотки в очень сильном поле. Целью настоящей работы является обоснование этого метода получения сверхсильного поля в неразрушаемом магните. Исходная модель магнитной системы с обмоткой малой толщины и диамагнитным экраном представлена на рис. 1. Обмотка может быть построена так, что магнитные давления полоидального поля в точках $M$ и

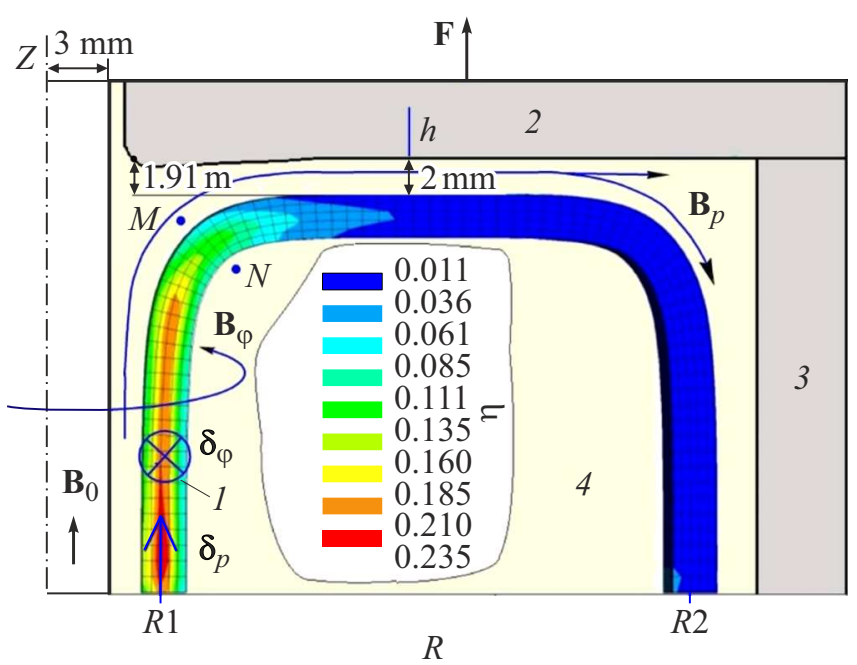

Рис. 1. Модель магнита с квазибессиловой обмоткой, торцевым и коаксиальным диамагнитными экранами и рассчитанное распределение нормированных напряжений. 1 - обмотка, 2 - торцевой экран, 3 - коаксиальный экран, 4 - диэлектрическая среда. 
азимутального в точках $N$ равны. При этом на границе обмотки отсутствует нормальная компонента индукции полоидального поля. В такой обмотке имеет место локальное равновесие проводников, и ожидаемые значения механических напряжений могут быть существенно ниже магнитного давления $P_{0}[3,4]$. В основной части обмотки 1 витки размещены под углом к оси, близким к $45^{\circ}$. При размещении вблизи торцевой части обмотки 2 идеально проводящего диска (экрана) 3 может быть обеспечено равновесие не только основной (цилиндрической), но и торцевой части обмотки. В приближении идеальной проводимости локальное равновесие тонкостенной обмотки может иметь место как на плоской части торца, так и на переходном участке определенной конфигурации, если внутренний радиус обмотки $R_{1}$ и зазор $h$ между обмоткой и диском связаны условием $h=0.5 R_{1}$ [5]. Экран подвержен воздействию аксиальной силы. При постоянной толщине зазора индукция полоидального поля в зазоре $B_{p}$ совпадает с радиальной компонентой индукции $B_{r}: B_{p}=B_{r}=B_{0} R_{1} / r$. В этом случае аксиальная сила определяется как

$$
F_{z} \approx \int_{R_{1}}^{R_{2}} \frac{B_{r}^{2} \pi r d r}{\mu_{0}}=\frac{B_{0}^{2} \pi R_{1}^{2}}{\mu_{0}} \ln A .
$$

При кратковременном воздействии поля импульс этой силы $P=\int_{0}^{\infty} F_{z} d t$ может быть достаточно мал, что позволяет обеспечить инерционное удержание диска и разгрузку торцевой части. Указанное можно подтвердить оценкой, выполненной в модели магнита с обмоткой нулевой толщины, когда сила $F_{z}$, рассчитанная без учета краевых эффектов, может быть представлена формулой (1). В том случае, когда индукция $B_{0}$ имеет вид униполярного импульса в форме полуволны синусоиды с амплитудой $B_{m}$ и полупериодом $\tau$, смещение диска с массой $m$ к концу импульса может быть рассчитано по формуле

$$
\Delta z=\frac{B_{m}^{2} \pi R_{1}^{2} \ln A}{\mu_{0} m} \int_{0}^{\tau} d t \int_{0}^{t} \sin ^{2}\left(\frac{\pi t}{\tau}\right) d t=\frac{B_{m}^{2} \pi R_{1}^{2} \tau^{2} \ln A}{4 \mu_{0} m} .
$$

Даже при амплитуде индукции $100 \mathrm{~T}$ для магнита малого объема с радиусами $R_{1}=5 \mathrm{~mm}, R_{2}=40 \mathrm{~mm}$ при $\tau=50 \mu$ s получаем, что тело с массой $3 \mathrm{~kg}$ к моменту максимума импульса смещается лишь на расстояние $0.27 \mathrm{~mm}$. Это дает основание считать при оценках неизменной конфигурацию магнитной системы в течение разряда.

Важным моментом при построении обмотки является учет реальной конфигурации линий тока. Отношение длины обмотки к внутреннему радиусу и внешний радиус торцевой части должны быть выбраны так, чтобы азимутальные координаты начала и конца каждой секции совпадали. Это обеспечивает возможность их последо-

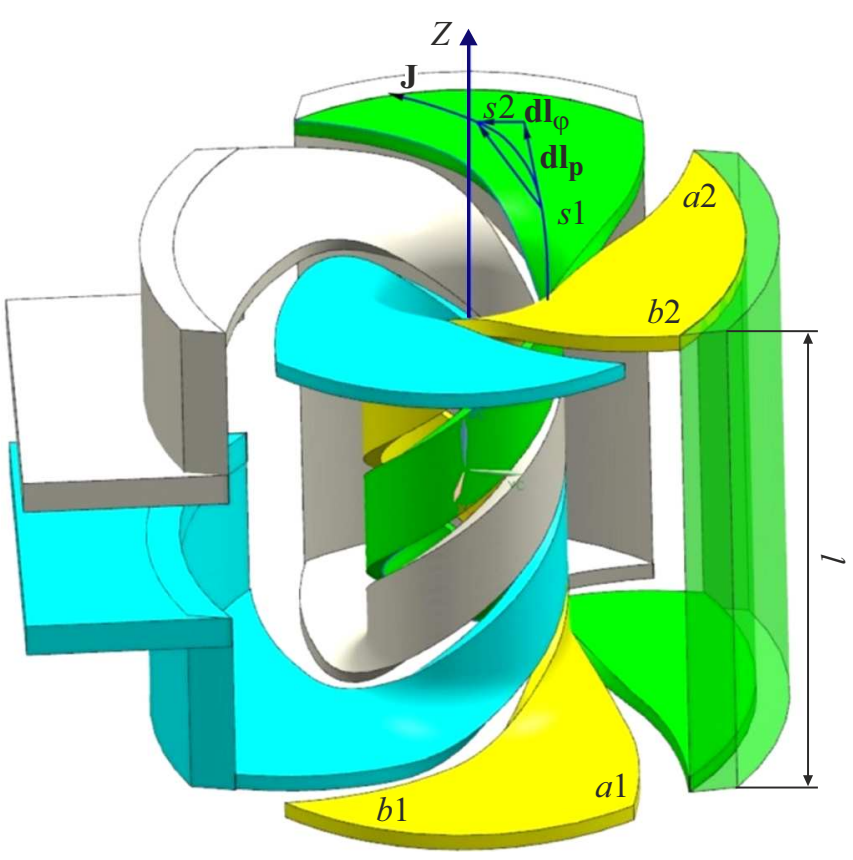

Рис. 2. Конфигурация обмотки с последовательным соединением ее четырех частей.

вательного соединения, как это показано на рис. $2{ }^{1}$ Расположение выводов определяется конфигурацией линий тока. В модели обмотки малой толщины компоненты смещения $d l_{p}$ и $d l_{\varphi}$ соотносятся так же, как компоненты плотности тока:

$$
\frac{d l_{\varphi}}{d l_{p}}=\frac{H_{p}}{H_{\varphi}}=\operatorname{tg} \alpha
$$

При изменении радиальной координаты точки $s$ на этой линии от значения $R_{1}$ до значения $R_{2}$ азимутальная координата получает приращение

$$
\Delta \varphi=\int_{R_{1}}^{R_{2}} \frac{H_{p} d l_{p}}{H_{\varphi} r}
$$

Линии тока, построенные по этому закону при условии $H_{p}=H_{\varphi}$, являются границами изоляционных зазоров между витками обмотки, показанной на рис. 2. В центральной части магнита $d l_{p}=d z$ эти линии являются спиралями: $\Delta \varphi=\Delta z$. На плоской части торца $d l_{p}=d r$, и линии тока описываются уравнением логарифмической спирали

$$
\Delta \varphi=\int_{R_{1}}^{R_{2}} \frac{d r}{r}=\ln \frac{R_{2}}{R_{1}} .
$$

В представленной на рис. 2 модели магнита, состоящего из четырех последовательно включенных секций,

\footnotetext{
${ }^{1}$ На рис. 2 не показан один из проводников, соединяющих части обмотки, а также торцевой и коаксиальный экраны.
} 
отношение радиусов $R_{2} / R_{1}$ и отношение длины к радиуcy $l / R_{1}$ выбраны так, что точки $a 1$ и $b 1$ смещаются на угол $3 / 2 \pi$ при переходе в положения $a 2$ и $b 2$. Это обеспечивает возможность последовательного соединения витков. Толщина обмотки должна быть выбрана с учетом джоулева нагрева проводника в процессе диффузии импульсного магнитного поля. Дополнительным фактором, влияющим на выбор толщины, является требование, чтобы к моменту максимума тока распределение полоидального и азимутального токов в обмотке было близко к однородному. Лишь в этом случае происходит характерная для квазибессиловой обмотки частичная компенсация встречно направленных объемных лоренцевых сил, обусловленных этими токами. Таким образом, обмотка должна работать в условиях слабо выраженного скин-эффекта. Характерной особенностью квазибессиловой обмотки является слабая зависимость остаточных напряжений от ее толщины. Это позволяет выбрать толщину исходя из конструктивных и технологических соображений. Дальнейший анализ проведен для магнита с обмоткой из нержавеющей стали с внутренним радиусом $R_{1}=5 \mathrm{~mm}$ и толщиной стенки $d=3 \mathrm{~mm}$. В первом приближении расчет проводится без учета влияния скинэффекта на распределение плотности полоидального и азимутального токов в момент максимума индукции, когда механические напряжения максимальны. После окончательного выбора длительности импульса с учетом нагрева обмотки проводится контрольный расчет, учитывающий диффузию поля в ходе разряда.

Конфигурация магнита с обмоткой малой толщины использована как основа для построения его расчетной 2Dмодели с указанными размерами. Использовано допущение об идеальной проводимости экрана. При выбранной длительности импульса это приближение приемлемо, если экран имеет покрытие из меди. В ходе расчетов с использованием программ Comsol-Multiphysics и Ansys произведена коррекция формы обмотки и изоляционного зазора между экраном и обмоткой с целью достижения минимального значения прочностного параметра - нормированного эквивалентного напряжения $\eta=2 \mu_{0} \sigma_{M} / B_{0}^{2}$, равного отношению этого напряжения, рассчитанного по формуле фон Мизеса, к магнитному давлению генерируемого поля. Значение $\eta_{\max }$ может быть снижено путем заполнения области, прилегающей к обмотке, диэлектрической средой с достаточно высоким модулем упругости. На рис. 1 показаны оптимизированные конфигурации экрана и обмотки, а также распределение интенсивности нормированных механических напряжений по Мизесу в теле обмотки. При использовании диэлектрической среды с модулем упругости $80 \mathrm{MPa}$ максимальное значение нормированного напряжения составляет $\eta_{\max }=0.235$. Это соответствует максимальному механическому напряжению в обмотке $0.94 \mathrm{GPa}$ при значении индукции на оси магнита $100 \mathrm{~T}$.

Наряду с прочностью материала нагрев обмотки является фактором, лимитирующим достижимый уровень индукции. Приращение температуры за время разряда мо-

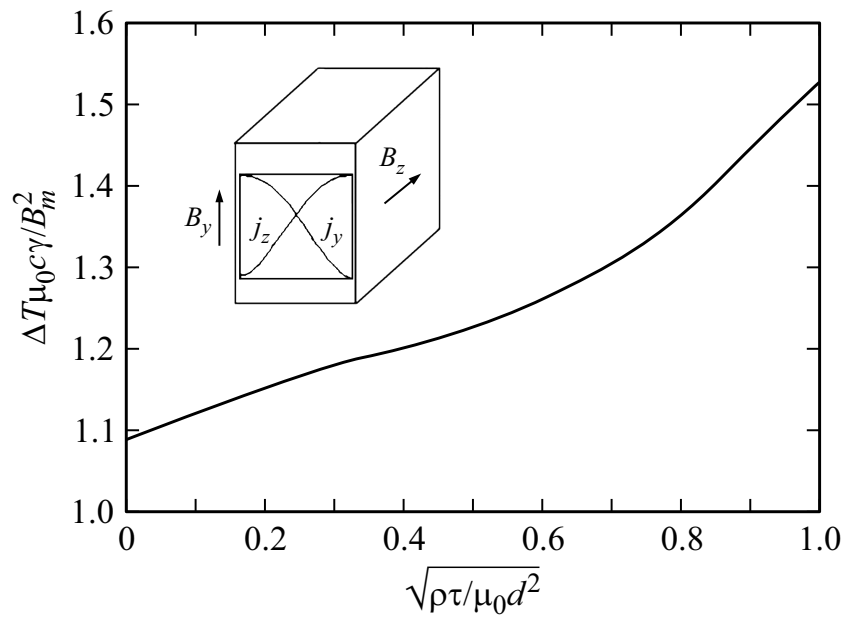

Рис. 3. Характерное распределение ортогональных магнитных полей и токов и нагрев поверхностей плоского слоя.

жет быть рассчитано по формуле $\Delta T=f(k) B_{m}^{2} /\left(\mu_{0} \gamma c\right)$, где $\gamma$ - плотность материала, $c-$ удельная теплоемкость, $B_{m}$ - амплитуда индукции импульсного поля, имеющего форму полупериода синусоиды, $f(k)$ - безразмерная функция параметра $k=\left(\rho \tau / \mu_{0}\right)^{1 / 2} / d$ (представлена на рис. 3), $\rho$ и $d-$ удельное электрическое сопротивление и толщина проводника соответственно. Анализ данных рис. 3 при амплитуде индукции $100 \mathrm{~T}$ показывает неприемлемо высокий нагрев проводника. Известным техническим решением снижения нагрева проводников в импульсном магнитном поле является применение проводящей среды с переменной по толщине электропроводностью [4]. При этом должны быть соблюдены условия более низкой проводимости поверхностных слоев проводника по сравнению с проводимостью в его глубине. Наибольший эффект достигается применением среды с плавно изменяющейся проводимостью: от низкой на поверхности к высокой в глубине [6]. Применительно к рассматриваемой здесь обмотке был исследован нагрев плоского проводника с параболическим распределением удельного электрического сопротивления по его толщине $\rho(x)=\left(\rho_{\max }-\rho_{\min }\right)(2 x / d-1)^{2}+\rho_{\min }$. При этом для толщины проводника $d=3 \mathrm{~mm}$ и $\rho_{\max }=0.224 \mu \Omega \cdot \mathrm{m}$, $\rho_{\min }=0.05 \mu \Omega \cdot \mathrm{m}$ полное приращение температуры (за время $t=2 \tau=100 \mu \mathrm{s})$ в поле с амплитудой индукции $100 \mathrm{~T}$ снижается до $1300^{\circ} \mathrm{C}$, что приемлемо для проводящих материалов, получаемых с помощью технологии селективного лазерного сплавления металлических порошков (SLM).

Таким образом, показано, что при получении коротких импульсов магнитного поля в магните малого объема можно избежать его разрушения благодаря применению диамагнитного экрана с инерционным удержанием. Подобная система может быть основой для создания неразрушаемых магнитов со сверхсильным полем. Для изготовления обмотки могут быть использованы совре- 
менные методы аддитивной технологии. При использовании диэлектрической среды с высоким модулем упругости механическая прочность обмотки может быть обеспечена в поле с индукцией до 100 Т. Нагрев обмотки, не превышающий допустимого предела, может иметь место в поле с индукцией до 70 Т, если проводимость постоянна по толщине витка. В поле с индукцией 100 Т допустимый нагрев возможен, если проводимость определенным образом изменяется по толщине проводника.

\section{Благодарности}

Результаты работы получены с использованием вычислительных ресурсов суперкомпьютерного центра СанктПетербургского политехнического университета Петра Великого (www.scc.spbstu.ru).

\section{Финансирование работы}

Работа выполнена при поддержке Российского научного фонда (грант № 18-19-00230).

\section{Конфликт интересов}

Авторы заявляют, что у них нет конфликта интересов.

\section{Список литературы}

[1] F. Herlach, Rep. Prog. Phys., 62 (6), 859 (1999). DOI: $10.1088 / 0034-4885 / 62 / 6 / 201$

[2] C.A. Swenson, W.S. Marshall, A.V. Gavrilin, K. Han, J. Schillig, J.R. Sims, H.J. Schneider-Muntau, Physica B, 346-347, 561 (2004). https://doi.org/10.1016/j.physb.2004.01.082

[3] Г.А. Шнеерсон, И.А. Вечеров, Д.А. Дегтев, О.С. Колтунов, С.И. Кривошеев, С.Л. Шишигин, ЖТФ, 78 (10), 29 (2008). [Пер. версия: 10.1134/S1063784208100046].

[4] G.A. Shneerson, M.I. Dolotenko, S.I. Krivosheev, Strong and superstrong pulsed magnetic fields generation (De Gruyter, Berlin, 2014). https://doi.org/10.1515/9783110252576

[5] G.A. Shneerson, O.S. Koltunov, H.J. Shneider-Muntau, V.V. Titkov, A.A. Parfentjev, Physica B, 346-347, 566 (2004). DOI: $10.1016 /$ j.physb.2004.01.083

[6] И.М. Карпова, В.В. Титков, Электричество, № 12, 55 (1999). 